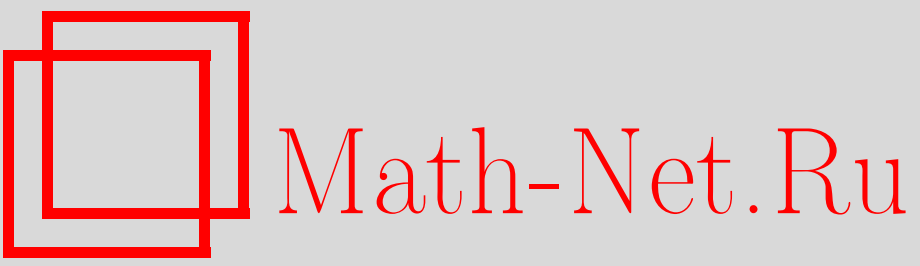

Н. М. Меженная, В. Г. Михайлов, Об асимптотической нормальности чисел появлений знаков в неравновероятной мультициклической случайной последовательности по модулю 4, Матем. вопр. криптогр., 2016, том 7, выпуск 4, 81-94

DOI: https://doi.org/10.4213/mvk205

Использование Общероссийского математического портала Math-Net.Ru подразумевает, что вы прочитали и согласны с пользовательским соглашением

http://www.mathnet.ru/rus/agreement

Параметры загрузки:

IP : 54.198 .67 .100

26 апреля 2023 г., 13:20:52 
МАТЕМАТИЧЕСКИЕ ВОПРОСЫ КРИПТОГРАФИИ

2016 T. 7 № 4 C. $81-94$

УДК 519.212.2+519.214

\title{
Об асимптотической нормальности чисел появлений знаков в неравновероятной мультициклической случайной последовательности по модулю 4
}

\author{
Н. М. Меженная ${ }^{1}$, В. Г. Михайлов ${ }^{2}$ \\ ${ }^{1}$ МГТУ им. Н. Э. Баумана, Москва \\ ${ }^{2}$ Математический институт им. В. А. Стеклова РАН, Москва \\ Получено 19.II.2016
}

Аннотация. Указаны условия асимптотической нормальности вектора частот появления знаков на цикле неравновероятной мультициклической случайной последовательности помодулю 4.

Научные исследования Н. М. Меженной поддержаны Российским фондом фундаментальных исследований, проект 14-01-00318а.

Ключевые слова: неравновероятная мультициклическая случайная последовательность, частоты знаков, асимптотическая нормальность

On the asymptotic normality of frequencies of values in the non-equiprobable multi-cyclic random sequence modulo 4

N. M. Mezhennaya ${ }^{1}$, V. G. Mikhailov ${ }^{2}$

${ }^{1}$ Bauman State Technical University, Moscow

${ }^{2}$ Steklov Mathematical Institute of Russian Academy of Sciences, Moscow

Abstract. For the frequencies of values on the cycle of the non-equiprobable multicyclic random sequence modulo 4 we obtain the conditions of asymptotic normality.

Keywords: non-equiprobable multi-cyclic random sequence, frequencies of values, asymptotic normality

Citation: Mathematical Aspects of Cryptography, 2016, v. 7, № 4, pp. 81-94 (Russian)

(c) Академия криптографии Российской Федерации, 2016 г. 


\section{1. Введение}

Пусть $n_{1}, \ldots, n_{r} \geqslant 2$ - взаимно простые натуральные числа. Мультициклическая случайная последовательность $\left\{Z_{t}\right\}_{t \geqslant 0}$ со значениями в $\{0, \ldots, M-1\}$ образуется по правилу $Z_{t}=\sum_{j=1}^{r} X_{t\left(n_{j}\right)}^{(j)} \bmod M, \quad t=0,1, \ldots$,
где $\left(X_{0}^{(j)}, \ldots, X_{n_{j}-1}^{(j)}\right), \quad j=1, \ldots, r,-$ независимые наборы независимых
случайных величин, принимающих значения в множестве наименьших неслучайных величин, принимающх воцельных вычетов по модулю $M$ (случайные величины с одним и тем же значением $j$ одинаково распределены), а $t\left(n_{j}\right)=t-\left[t / n_{j}\right] n_{j}$. Отрезок $Z(r)=\left(Z_{0}, Z_{1}, \ldots, Z_{n_{1} \cdots n_{r}-1}\right)$ будем называть ииклом мультициклической последовательности $\left\{Z_{t}\right\}$. Последовательности такого вида были введены в [1] в связи с исследованием одного генератора псевдослучайных чисел над полем $G F(2)$ (генератора Пола).

Свойства распределения числа единиц на цикле мультициклической последовательности над $G F(2)$ исследовались в работах [2-4].

Нас интересует случай $M=4$. Целью исследования является изучение асимптотического поведения при $n_{1}, n_{2}, \ldots, n_{r} \rightarrow \infty$ совместного распределения случайных величин $\varkappa_{a_{2} a_{1}}(r)$ - количеств чисел в последовательности $Z(r)$, имеющих двоичную запись $a_{2} a_{1}$, где $a_{1}, a_{2} \in\{0,1\}$.

Нетрудно показать (см. [5]), что система уравнений

$$
\varkappa_{a_{2} a_{1}}(r)=\frac{1}{4}\left(n_{1} n_{2} \cdots n_{r}+(-1)^{a_{1}} \beta(r)+2(-1)^{a_{2}} \beta_{a_{1}}(r)\right), \quad a_{1}, a_{2} \in\{0,1\},
$$

однозначно определяет величины $\beta(r), \beta_{0}(r), \beta_{1}(r)$ и сводит задачу исследования совместного распределения величин $\varkappa_{a_{2} a_{1}}(r)$ к изучению распределения вектора $\left(\beta(r), \beta_{0}(r), \beta_{1}(r)\right)^{T}$ (это вектор-столбец).

В [5] было показано, что если распределение знаков $X_{k}^{(j)}$ является равномерным, то при $n_{1} \cdots n_{r} \rightarrow \infty$ величина $\left(n_{1} \cdots n_{r}\right)^{-1 / 2} \beta(r)$ и вектор $2^{r / 2}\left(n_{1} \cdots n_{r}\right)^{-1 / 2}\left(\beta_{0}(r), \beta_{1}(r)\right)$ асимптотически независимы, распределение величины $\left(n_{1} \cdots n_{r}\right)^{-1 / 2} \beta(r)$ сходится к распределению произведения $r$ независимых стандартных нормальных случайных величин, а распределение вектора $2^{r / 2}\left(n_{1} \cdots n_{r}\right)^{-1 / 2}\left(\beta_{0}(r), \beta_{1}(r)\right)$ сходится к распределению вектора $\left(\varrho_{r} \cos \phi, \varrho_{r} \sin \phi\right)$, где случайная величина $\varrho_{r}$ представляется как произведение $r$ независимых случайных величин, имеющих распределение Рэлея с плотностью $f(x)=x e^{-x^{2} / 2}, x \geqslant 0$, а $\phi$ имеет равномерное распределение на $[0,2 \pi]$. 
Если же распределение знаков $X_{k}^{(j)}$ отличается от равномерного, то в типичных случаях при $n_{1} n_{2} \cdots n_{r} \rightarrow \infty$ и при подходящих центрировании и нормировке распределение вектора $\left(\beta(r), \beta_{0}(r), \beta_{1}(r)\right)$ сходится к трехмерному нормальному распределению. Опишем эти случаи.

Введем обозначения

$$
\begin{gathered}
p_{a_{2} a_{1}}^{(j)}=\mathbf{P}\left\{X_{k}^{(j)}=2 a_{2}+a_{1}\right\}, \quad a_{1}, a_{2} \in\{0,1\}, \\
b^{(j)}=\left(p_{00}^{(j)}+p_{10}^{(j)}\right)-\left(p_{01}^{(j)}+p_{11}^{(j)}\right), \quad b_{a}^{(j)}=p_{0 a}^{(j)}-p_{1 a}^{(j)}, \quad a \in\{0,1\},
\end{gathered}
$$

и определим матрицу $\mathbb{B}(r)$ и величины $b^{*}, b_{0}^{*}, b_{1}^{*}$ формулами

$$
B^{(j)}=\left(\begin{array}{ccc}
b^{(j)} & 0 & 0 \\
0 & b_{0}^{(j)} & -b_{1}^{(j)} \\
0 & b_{1}^{(j)} & b_{0}^{(j)}
\end{array}\right), \quad \mathbb{B}(r)=\left(\begin{array}{ccc}
b^{*} & 0 & 0 \\
0 & b_{0}^{*} & -b_{1}^{*} \\
0 & b_{1}^{*} & b_{0}^{*}
\end{array}\right)=\prod_{j=1}^{r} B^{(j)} .
$$

Теорема 1. Пусть $r \geqslant 2$. Тогда

$$
\left(\begin{array}{ccc}
\mathbf{E} \beta(r) & 0 & 0 \\
0 & \mathbf{E} \beta_{0}(r) & -\mathbf{E} \beta_{1}(r) \\
0 & \mathbf{E} \beta_{1}(r) & \mathbf{E} \beta_{0}(r)
\end{array}\right)=n_{1} n_{2} \cdots n_{r} \mathbb{B}(r)
$$

Следствие 1. Пусть $r \geqslant 2$. Тогда

$$
\mathbf{E}\left(\beta(r), \mathbf{E} \beta_{0}(r), \mathbf{E} \beta_{1}(r)\right)^{T}=n_{1} n_{2} \cdots n_{r}\left(b^{*}, b_{0}^{*}, b_{1}^{*}\right)^{T}
$$

Введем обозначения $\widetilde{\Sigma}^{(j)}=$

$$
=\left(\begin{array}{ccc}
p_{0}^{(j)} q_{0}^{(j)} & p_{00}^{(j)} q_{00}^{(j)}-p_{10}^{(j)} q_{10}^{(j)} & p_{0}^{(j)}\left(p_{11}^{(j)}-p_{01}^{(j)}\right) \\
p_{00}^{(j)} q_{00}^{(j)}-p_{10}^{(j)} q_{10}^{(j)} & p_{0}^{(j)}-\left(p_{00}^{(j)}-p_{10}^{(j)}\right)^{2} & -\left(p_{00}^{(j)}-p_{10}^{(j)}\right)\left(p_{01}^{(j)}-p_{11}^{(j)}\right) \\
p_{0}^{(j)}\left(p_{11}^{(j)}-p_{01}^{(j)}\right) & -\left(p_{00}^{(j)}-p_{10}^{(j)}\right)\left(p_{01}^{(j)}-p_{11}^{(j)}\right) & p_{1}^{(j)}-\left(p_{01}^{(j)}-p_{11}^{(j)}\right)^{2}
\end{array}\right),
$$

где

$$
q_{00}^{(j)}=1-p_{00}^{(j)}, \quad q_{10}^{(j)}=1-p_{10}^{(j)}, \quad q_{0}^{(j)}=1-p_{0}^{(j)},
$$

и обозначения

$$
V^{j}=\left(\begin{array}{ccc}
v^{(j)} & 0 & 0 \\
0 & v_{0}^{(j)} & -v_{1}^{(j)} \\
0 & v_{1}^{(j} & v_{0}^{(j)}
\end{array}\right), \quad j=1, \ldots, r
$$


где

$$
v^{(j)}=\frac{b^{*}}{b^{(j)}}, \quad v_{0}^{(j)}=\frac{b_{0}^{*} b_{0}^{(j)}+b_{1}^{*} b_{1}^{(j)}}{\left(b_{0}^{(j)}\right)^{2}+\left(b_{1}^{(j)}\right)^{2}}, \quad v_{1}^{(j)}=\frac{b_{1}^{*} b_{0}^{(j)}-b_{0}^{*} b_{1}^{(j)}}{\left(b_{0}^{(j)}\right)^{2}+\left(b_{1}^{(j)}\right)^{2}}
$$

Теорема 2. Пусть $r \geqslant 2$, числа $n_{1}<\cdots<n_{r}$ стремятся $\kappa$ бесконечности, причем существуют предель $\lim n_{1} / n_{j}=\rho_{j}^{2} \in(0,1], j=1, \ldots, r$. Пусть

$$
\operatorname{det} B^{(j)}>0, \quad j=1, \ldots, r .
$$

Тогда распределение вектора

$$
\nabla(r)=n_{1}^{1 / 2}\left(\frac{1}{n_{1} n_{2} \cdots n_{r}}\left(\begin{array}{c}
\beta(r) \\
\beta_{0}(r) \\
\beta_{1}(r)
\end{array}\right)-\left(\begin{array}{c}
b^{*} \\
b_{0}^{*} \\
b_{1}^{*}
\end{array}\right)\right)
$$

сходится к трехмерному нормальному распределению с нулевым вектором средних и матрицей ковариащий

$$
\Sigma(r)=\sum_{j=1}^{r} \rho_{j}^{2} V^{(j)} \widetilde{\Sigma}^{(j)}\left(V^{(j)}\right)^{T}
$$

Следствие 2. Пусть выполнены условия теоремы 2 и

$$
p_{a b}^{(1)}=\cdots=p_{a b}^{(r)}, \quad a, b \in\{0,1\}
$$

Тогда распределение вектора $\nabla(r)$ сходится к трехмерному нормальному распределению с нулевым вектором средних и матрищей ковариаций

$$
\Sigma(r)=\left(\sum_{j=1}^{r} \rho_{j}^{2}\right) \cdot V^{(1)} \widetilde{\Sigma}^{(1)}\left(V^{(1)}\right)^{T}
$$

Замечание 1. Условие (6) предполагает, что $\min _{j=1, \ldots, r} b^{(j)}>0$. Последнее условие является необходимым условием для асимптотической нормальности случайной величины $\beta(r)$ и вектора $\nabla(r)$ в целом. При его нарушении в качестве предельных распределений выступают иные распределения. Подробности можно найти в [3], где был рассмотрен случай, когда $M=2$. 


\section{2. Вспомогательные утверждения}

Пусть $X_{1}, \ldots, X_{n}$ - последовательность независимых одинаково распределенных случайных величин, принимающих значения из множества $\{0,1,2,3\}$ и и

$$
p_{a b}=\mathbf{P}\left\{X_{k}=2 a_{2}+a_{1}\right\}, \quad a_{1}, a_{2} \in\{0,1\} .
$$

Будем использовать обозначения

$$
p_{a}=p_{0 a}+p_{1 a}, \quad p_{a_{2} a_{1}}=1-q_{a_{2} a_{1}}, \quad a, a_{1}, a_{2} \in\{0,1\} .
$$

Пусть $\nu_{a_{2} a_{1}}=\nu_{a_{2} a_{1}}(n)$ - количество чисел в $X_{1}, \ldots, X_{n}$, имеющих двоичную запись $a_{2} a_{1}$, и $\nu_{a}=\nu_{a}(n)-$ количество чисел в $X_{1}, \ldots, X_{n}$, двоичная запись которых заканчивается числом $a$.

Положим

$$
\begin{aligned}
& \delta=\nu_{0}-\nu_{1}, \quad \delta_{0}=\nu_{00}-\nu_{10}, \quad \delta_{1}=\nu_{01}-\nu_{11}, \\
& b=p_{0}-p_{1}, \quad b_{0}=p_{00}-p_{10}, \quad b_{1}=p_{01}-p_{11} .
\end{aligned}
$$

Тогда

$$
\mathbf{E} \delta=n b, \quad \mathbf{E} \delta_{0}=n b_{0}, \quad \mathbf{E} \delta_{1}=n b_{1} .
$$

Введем случайные величины $\theta=\delta-n b, \theta_{0}=\delta_{0}-n b_{0}, \theta_{1}=\delta_{1}-n b_{1}$ и рассмотрим вектор-столбцы

$$
\Theta=\left(\theta, \theta_{0}, \theta_{1}\right)^{T}, \quad \widetilde{\Theta}=\frac{1}{\sqrt{n}} \Theta .
$$

Лемма 1. Пусть $n \rightarrow \infty$. Тогда распределение вектора $\widetilde{\Theta}$ сходится к трехмерному нормальному распределению с нулевым вектором средних и ковариационной матрицей

$$
\widetilde{\Sigma}=\left(\begin{array}{ccc}
p_{0} p_{1} & p_{00} q_{00}-p_{10} q_{10} & p_{0}\left(p_{11}-p_{01}\right) \\
p_{00} q_{00}-p_{10} q_{10} & p_{0}-\left(p_{00}-p_{10}\right)^{2} & -\left(p_{00}-p_{10}\right)\left(p_{01}-p_{11}\right) \\
p_{0}\left(p_{11}-p_{01}\right) & -\left(p_{00}-p_{10}\right)\left(p_{01}-p_{11}\right) & p_{1}-\left(p_{01}-p_{11}\right)^{2}
\end{array}\right) .
$$

Замечание 2. Из леммы 1 следует, что при $n \rightarrow \infty$ выполнены соотношения $\theta=O\left(n^{1 / 2}\right), \theta_{0}=O\left(n^{1 / 2}\right), \theta_{1}=O\left(n^{1 / 2}\right)$. Здесь и далее запись $\xi=O(f(n))$ означает, что случайная величина $\xi / f(n)$ при $n \rightarrow \infty$ ограничена по вероятности. 
Доказательство. Так как вектор $\widetilde{\nu}=\left(\nu_{00}, \nu_{01}, \nu_{10}, \nu_{11}\right)^{T}$ имеет мультиномиальный закон распределения с параметрами $n$ и $\left\{p_{00}, p_{01}, p_{10}, p_{00}\right\}$, то его вектор средних и ковариационная матрица равны соответственно

$$
\mathbf{E} \widetilde{\nu}=\left(\begin{array}{c}
n p_{00} \\
n p_{01} \\
n p_{10} \\
n p_{11}
\end{array}\right), \quad \Sigma_{\widetilde{\nu}}=\left(\begin{array}{cccc}
n p_{00} q_{00} & -n p_{00} p_{01} & -n p_{00} p_{10} & -n p_{00} p_{11} \\
-n p_{00} p_{01} & n p_{01} q_{01} & -n p_{01} p_{10} & -n p_{01} p_{11} \\
-n p_{00} p_{10} & -n p_{01} p_{10} & n p_{10} q_{10} & -n p_{10} p_{11} \\
-n p_{00} p_{11} & -n p_{01} p_{11} & -n p_{10} p_{11} & n p_{11} q_{11}
\end{array}\right)
$$

Вектор $\Theta$ получен из вектора $\widetilde{\nu}$ в результате линейного преобразования

$$
\Theta=C \widetilde{\nu}-\left(b, b_{0}, b_{1}\right)^{T}, \quad C=\left(\begin{array}{cccc}
1 & 0 & 1 & 0 \\
1 & 0 & -1 & 0 \\
0 & 1 & 0 & -1
\end{array}\right) .
$$

Вектор средних и ковариационная матрица вектора $\Theta$ выражаются формулами

$$
\begin{gathered}
\mathbf{E} \Theta=\left(\begin{array}{cccc}
1 & 0 & 1 & 0 \\
1 & 0 & -1 & 0 \\
0 & 1 & 0 & -1
\end{array}\right) \mathbf{E} \widetilde{\nu}-\left(b, b_{0}, b_{1}\right)^{T}=\mathbf{0} \\
\Sigma_{\Theta}=C \Sigma_{\widetilde{\nu}} C^{T}= \\
=n\left(\begin{array}{ccc}
p_{0} p_{1} & p_{00} q_{00}-p_{10} q_{10} & p_{0}\left(p_{11}-p_{01}\right) \\
p_{00} q_{00}-p_{10} q_{10} & p_{0}-\left(p_{00}-p_{10}\right)^{2} & -\left(p_{00}-p_{10}\right)\left(p_{01}-p_{11}\right) \\
p_{0}\left(p_{11}-p_{01}\right) & -\left(p_{00}-p_{10}\right)\left(p_{01}-p_{11}\right) & p_{1}-\left(p_{01}-p_{11}\right)^{2}
\end{array}\right) .
\end{gathered}
$$

Из последней формулы и определения вектора $\widetilde{\Theta}$ следует, что ковариационная матрица вектора $\widetilde{\Theta}$ равна $\widetilde{\Sigma}$.

Далее понадобится следующее утверждение.

Лемма 2. Характеристическая функичия вектора $\widetilde{\Theta}$ имеет вид

$$
\begin{aligned}
g_{\widetilde{\Theta}}\left(t, t_{0}, t_{1}\right)= & \exp \left\{-\frac{i}{\sqrt{n}}\left(b t+b_{0} t_{0}+b_{1} t_{1}\right)\right\} \times \\
& \times\left(p_{01} e^{i \frac{t_{1}}{\sqrt{n}}}+p_{11} e^{-i \frac{t_{1}}{\sqrt{n}}}+e^{i \frac{t}{\sqrt{n}}}\left(p_{00} e^{i \frac{t_{0}}{\sqrt{n}}}+p_{10} e^{-i \frac{t_{0}}{\sqrt{n}}}\right)\right)^{n} .
\end{aligned}
$$


Доказательство. Сначала заметим, что

$$
\begin{aligned}
& \mathbf{P}\left\{\nu_{0}=k, \nu_{00}-\nu_{10}=l_{0}, \nu_{01}-\nu_{11}=l_{1}\right\}= \\
& =\mathbf{P}\left\{\nu_{00}=\frac{k+l_{0}}{2}, \nu_{10}=\frac{k-l_{0}}{2}, \nu_{01}=\frac{n-k+l_{1}}{2}, \nu_{11}=\frac{n-k+l_{1}}{2}\right\}= \\
& =C_{n}^{\frac{k+l_{0}}{2}} p_{00}^{\frac{k+l_{0}}{2}} C_{n-\frac{k+l_{0}}{2}}^{\frac{k-l_{0}}{2}} p_{10}^{\frac{k-l_{0}}{2}} C_{n-k}^{\frac{n-k+l_{1}}{2}} p_{01}^{\frac{n-k+l_{1}}{2}} p_{11}^{\frac{n-k-l_{1}}{2}},
\end{aligned}
$$

где $C_{m}^{j}=0$, если $j$ или $m$ не является целым. Сделаем замену

$$
k_{0}=\frac{k+l_{0}}{2}, \quad k_{1}=\frac{n-k+l_{1}}{2} .
$$

Тогда характеристическая функция вектора $\nu=\left(\nu_{0}, \nu_{00}-\nu_{10}, \nu_{10}-\nu_{11}\right)^{T}$ равна

$$
\begin{aligned}
& g_{\nu}\left(t, t_{0}, t_{1}\right)=\mathbf{E} e^{i\left(t \nu_{0}+t_{0}\left(\nu_{00}-\nu_{10}\right)+t_{1}\left(\nu_{01}-\nu_{11}\right)\right)}= \\
& =\sum_{k=0}^{n} \sum_{k_{0}=0}^{k} \sum_{k_{1}=0}^{n-k} C_{n}^{k_{0}} p_{00}^{k_{0}} C_{n-k_{0}}^{k-k_{0}} p_{10}^{k-k_{0}} C_{n-k}^{k_{1}} p_{01}^{k_{1}} p_{11}^{n-k-k_{1}} e^{i t_{0}\left(2 k_{0}-k\right)} e^{i t_{1}\left(2 k_{1}-(n-k)\right)} e^{i t k} .
\end{aligned}
$$

Так как

$$
\begin{aligned}
& \sum_{k_{1}=0}^{n-k} C_{n-k}^{k_{1}} p_{01}^{k_{1}} p_{11}^{n-k-k_{1}} e^{i t_{1}\left(2 k_{1}-(n-k)\right)}= \\
& =e^{-i t_{1}(n-k)}\left(p_{01} e^{2 i t_{1}}+p_{11}\right)^{n-k}=\left(p_{01} e^{i t_{1}}+p_{11} e^{-i t_{1}}\right)^{n-k}, \\
& \sum_{k_{0}=0}^{k} C_{n}^{k_{0}} p_{00}^{k_{0}} C_{n-k_{0}}^{k-k_{0}} p_{10}^{k-k_{0}} e^{i t_{0}\left(2 k_{0}-k\right)}= \\
& =e^{-i t_{0} k} \sum_{k_{0}=0}^{k} \frac{k !}{k_{0} !\left(k-k_{0}\right) !} \frac{n !}{k !(n-k) !} p_{00}^{k_{0}} p_{10}^{k-k_{0}} e^{2 i t_{0} k_{0}}= \\
& =C_{n}^{k} e^{-i t_{0} k}\left(p_{00} e^{2 i t_{0} k}+p_{10}\right)^{k}=C_{n}^{k}\left(p_{00} e^{i t_{0}}+p_{10} e^{-i t_{0}}\right)^{k},
\end{aligned}
$$


TO

$$
\begin{aligned}
g_{\nu}\left(t, t_{0}, t_{1}\right) & =\sum_{k=0}^{n} C_{n}^{k}\left(p_{00} e^{i t_{0}}+p_{10} e^{-i t_{0}}\right)^{k}\left(p_{01} e^{i t_{1}}+p_{11} e^{-i t_{1}}\right)^{n-k} e^{i t}= \\
& =\left(p_{01} e^{i t_{1}}+p_{11} e^{-i t_{1}}+e^{i t}\left(p_{00} e^{i t_{0}}+p_{10} e^{-i t_{0}}\right)\right)^{n}
\end{aligned}
$$

Теперь рассмотрим вектор $\widetilde{\Theta}$. Так как

$$
\widetilde{\Theta}=\frac{1}{\sqrt{n}}\left(\nu-\left(b, b_{0}, b_{1}\right)^{T}\right)
$$

то из (10) получаем (9):

$$
\begin{aligned}
g_{\widetilde{\Theta}}\left(t, t_{0}, t_{1}\right)= & \exp \left\{-\frac{i}{\sqrt{n}}\left(b t+b_{0} t_{0}+b_{1} t_{1}\right)\right\} g_{\nu}\left(\frac{t}{\sqrt{n}}, \frac{t_{0}}{\sqrt{n}}, \frac{t_{1}}{\sqrt{n}}\right)= \\
= & \exp \left\{-\frac{i}{\sqrt{n}}\left(b t+b_{0} t_{0}+b_{1} t_{1}\right)\right\} \times \\
& \times\left(p_{01} e^{i \frac{t_{1}}{\sqrt{n}}}+p_{11} e^{-i \frac{t_{1}}{\sqrt{n}}}+e^{i \frac{t}{\sqrt{n}}}\left(p_{00} e^{i \frac{t_{0}}{\sqrt{n}}}+p_{10} e^{-i \frac{t_{0}}{\sqrt{n}}}\right)\right)^{n} .
\end{aligned}
$$

Лемма 2 доказана.

Вернемся к доказательству леммы 1. Разложим в ряд логарифм характеристической функции (9):

$$
\begin{aligned}
\ln g_{\widetilde{\Theta}}\left(t, t_{0}, t_{1}\right)= & -i \sqrt{n}\left(p_{0} t+\left(p_{00}-p_{10}\right) t_{0}+\left(p_{01}-p_{11}\right) t_{1}\right)+ \\
& +n g_{1}\left(t, t_{0}, t_{1}\right)
\end{aligned}
$$

где

$$
g_{1}\left(t, t_{0}, t_{1}\right)=\ln \left(p_{01} e^{i \frac{t_{1}}{\sqrt{n}}}+p_{11} e^{-i \frac{t_{1}}{\sqrt{n}}}+e^{i \frac{t}{\sqrt{n}}}\left(p_{00} e^{i \frac{t_{0}}{\sqrt{n}}}+p_{10} e^{-i \frac{t_{0}}{\sqrt{n}}}\right)\right)
$$

Так как

$$
e^{i \frac{u}{\sqrt{n}}}=1+i \frac{u}{\sqrt{n}}-\frac{u^{2}}{2 n}+O\left(n^{-3 / 2}\right), \quad n \rightarrow \infty
$$


To

$$
\begin{aligned}
g_{1}\left(t, t_{0}, t_{1}\right)=\ln \{ & p_{1}+p_{01}\left(i \frac{t_{1}}{\sqrt{n}}-\frac{t_{1}^{2}}{2 n}\right)-p_{11}\left(i \frac{t_{1}}{\sqrt{n}}+\frac{t_{1}^{2}}{2 n}\right)+ \\
& +\left(1+\frac{i t}{\sqrt{n}}-\frac{t^{2}}{2 n}\right)\left(p_{0}+p_{00}\left(\frac{i t_{0}}{\sqrt{n}}-\frac{t_{0}^{2}}{2 n}\right)-p_{10}\left(\frac{i t_{0}}{\sqrt{n}}+\frac{t_{0}^{2}}{2 n}\right)\right)+ \\
& \left.+O\left(n^{-3 / 2}\right)\right\}= \\
=\ln \{1 & +i \frac{t_{1}}{\sqrt{n}}\left(p_{01}-p_{11}\right)+i \frac{t_{0}}{\sqrt{n}}\left(p_{00}-p_{10}\right)+ \\
& +\frac{t}{\sqrt{n}}\left(i p_{0}-\frac{t_{0}}{\sqrt{n}}\left(p_{00}-p_{10}\right)\right)- \\
& \left.-\frac{t_{1}^{2}}{2 n} p_{1}-\frac{t_{0}^{2}}{2 n} p_{0}-\frac{t^{2}}{2 n} p_{0}+O\left(n^{-3 / 2}\right)\right\}
\end{aligned}
$$

Теперь воспользуемся разложением в ряд для логарифма и получим

$$
\begin{aligned}
g_{1}\left(t, t_{0}, t_{1}\right)= & i \frac{t_{1}}{\sqrt{n}}\left(p_{01}-p_{11}\right)+i \frac{t_{0}}{\sqrt{n}}\left(p_{00}-p_{10}\right)+ \\
+ & \frac{t}{\sqrt{n}}\left(i p_{0}-\frac{t_{0}}{\sqrt{n}}\left(p_{00}-p_{10}\right)\right)-\frac{t_{1}^{2}}{2 n} p_{1}-\frac{t^{2}}{2 n} p_{0}-\frac{t_{0}^{2}}{2 n} p_{0}- \\
& -\frac{1}{2}\left(i \frac{t_{1}}{\sqrt{n}}\left(p_{01}-p_{11}\right)+i \frac{t_{0}}{\sqrt{n}}\left(p_{00}-p_{10}\right)+i p_{0} \frac{t}{\sqrt{n}}\right)^{2}+ \\
+ & O\left(n^{-3 / 2}\right)= \\
= & i \frac{t_{1}}{\sqrt{n}}\left(p_{01}-p_{11}\right)+i \frac{t_{0}}{\sqrt{n}}\left(p_{00}-p_{10}\right)+i p_{0} \frac{t}{\sqrt{n}}- \\
- & \frac{1}{2 n}\left\{\left(p_{0}-p_{0}^{2}\right) t^{2}+\left(p_{1}-\left(p_{01}-p_{11}\right)^{2}\right) t_{1}^{2}+\right. \\
& +\left(p_{0}-\left(p_{00}-p_{10}\right)^{2}\right) t_{0}^{2}+ \\
& +2 t t_{0}\left(1-p_{0}\right)\left(p_{00}-p_{10}\right)-2 t t_{1} p_{0}\left(p_{01}-p_{11}\right)- \\
& \left.\quad-2 t_{0} t_{1}\left(p_{01}-p_{11}\right)\left(p_{00}-p_{10}\right)\right\}+ \\
+ & O\left(n^{-3 / 2}\right) .
\end{aligned}
$$

2016, T. 7, № 4, С. 81-94 
Подставляя это выражение для $g_{1}\left(t, t_{0}, t_{1}\right)$ в правую часть формулы $(11)$, с учетом равенства

$$
\left(1-p_{0}\right)\left(p_{00}-p_{10}\right)=p_{00} q_{00}-p_{10} q_{10}
$$

получаем, что

$$
\begin{aligned}
& \ln g_{\widetilde{\Theta}}\left(t, t_{0}, t_{1}\right)=-\frac{1}{2}\{\left(p_{0}-p_{0}^{2}\right) t^{2}+ \\
&+\left(p_{1}-\left(p_{01}-p_{11}\right)^{2}\right) t_{1}^{2}+\left(p_{0}-\left(p_{00}-p_{10}\right)^{2}\right) t_{0}^{2}+ \\
&+2 t t_{0}\left(p_{00} q_{00}-p_{10} q_{10}\right)-2 t t_{1} p_{0}\left(p_{01}-p_{11}\right)- \\
&\left.-2 t_{0} t_{1}\left(p_{01}-p_{11}\right)\left(p_{00}-p_{10}\right)\right\}+O\left(n^{-1 / 2}\right)= \\
&=-\frac{1}{2}\left(t, t_{0}, t_{1}\right) \widetilde{\Sigma}\left(t, t_{0}, t_{1}\right)^{T}+O\left(n^{-1 / 2}\right), \quad n \rightarrow \infty .
\end{aligned}
$$

Значит, распределение вектора $\widetilde{\Theta}$ сходится к трехмерному нормальному распределению с нулевым вектором средних и ковариационной матрицей $\widetilde{\Sigma}$. Лемма 1 доказана.

\section{3. Доказательства теорем}

Пусть $\nu_{a_{2} a_{1}}^{(j)}-$ количество чисел в $X^{(j)}=\left(X_{0}^{(j)}, \ldots, X_{n_{j}-1}^{(j)}\right)$, имеющих в двоичной системе счисления запись $a_{2} a_{1}$, и $\nu_{a_{1}}^{(j)}-$ количество чисел в $X^{(j)}$, двоичная запись которых заканчивается числом $a_{1}$. Положим

$$
\begin{gathered}
\delta^{(j)}=\nu_{0}^{(i)}-\nu_{1}^{(j)}, \quad \delta_{a}^{(j)}=\nu_{0 a}^{(j)}-\nu_{1 a}^{(j)}, \quad a \in\{0,1\}, \quad j=1, \ldots, r . \\
b^{(j)}=p_{0}^{(j)}-p_{1}^{(j)}, \quad b_{0}^{(j)}=p_{00}^{(j)}-p_{10}^{(j)}, \quad b_{1}^{(j)}=p_{01}^{(j)}-p_{11}^{(j)} .
\end{gathered}
$$

Тогда

$$
\mathbf{E} \delta^{(j)}=n_{j} b^{(j)}, \quad \mathbf{E} \delta_{0}^{(j)}=n_{j} b_{0}^{(j)}, \quad \mathbf{E} \delta_{1}^{(j)}=n_{j} b_{1}^{(j)} .
$$

Введем случайные величины

$$
\begin{gathered}
\theta^{(j)}=\delta^{(j)}-n_{j} b^{(j)}, \quad \theta_{0}^{(j)}=\delta_{0}^{(j)}-n_{j} b_{0}^{(j)}, \quad \theta_{1}^{(j)}=\delta_{1}^{(j)}-n_{j} b_{1}^{(j)}, \\
\widetilde{\theta}^{(j)}=\frac{1}{\sqrt{n}} \theta^{(j)}, \quad \widetilde{\theta}_{0}^{(j)}=\frac{1}{\sqrt{n}} \theta_{0}^{(j)}, \quad \widetilde{\theta}_{1}^{(j)}=\frac{1}{\sqrt{n}} \theta_{1}^{(j)}
\end{gathered}
$$


и рассмотрим вектор-столбцы

$$
\Theta^{(j)}=\left(\theta^{(j)}, \theta_{0}^{(j)}, \theta_{1}^{(j)}\right)^{T}, \quad \widetilde{\Theta}^{(j)}=\left(\widetilde{\theta}^{(j)}, \widetilde{\theta}_{0}^{(j)}, \widetilde{\theta}_{1}^{(j)}\right)^{T}
$$

В формуле (1) (подробности см. в [5])

$$
\left(\begin{array}{ccc}
\beta(r) & 0 & 0 \\
0 & \beta_{0}(r) & -\beta_{1}(r) \\
0 & \beta_{1}(r) & \beta_{0}(r)
\end{array}\right)=\prod_{j=1}^{r}\left(\begin{array}{ccc}
\delta^{(j)} & 0 & 0 \\
0 & \delta_{0}^{(j)} & -\delta_{1}^{(j)} \\
0 & \delta_{1}^{(j)} & \delta_{0}^{(j)}
\end{array}\right)
$$

Согласно (14) искомая формула может быть получена из двух аналогичных формул: одна для величины $\beta(r)$, а другая для вектора $\left(\beta_{0}(r), \beta_{1}(r)\right)^{T}$.

По условиям теорем все $b^{(j)}>0$. Поэтому согласно (14)

$$
\beta(r)=\prod_{j=1}^{r}\left(n_{j} b^{(j)}+\sqrt{n_{j}} \widetilde{\theta}^{(j)}\right)=\prod_{j=1}^{r} n_{j} b^{(j)}\left(1+\sum_{k=1}^{r} \frac{1}{\sqrt{n_{k}}} \frac{\widetilde{\theta}^{(k)}}{b^{(k)}}+w\right),
$$

где

$$
w=\sum_{l=2}^{r} \sum_{1 \leqslant k_{1}<k_{2}<\cdots<k_{l} \leqslant r} \frac{1}{\sqrt{n_{k_{1}} n_{k_{2}} \cdots n_{k_{l}}}} \frac{\widetilde{\theta}^{\left(k_{1}\right)} \widetilde{\theta}^{\left(k_{2}\right)} \cdots \widetilde{\theta}^{\left(k_{l}\right)}}{b^{\left(k_{1}\right) b^{\left(k_{2}\right)} \cdots b^{\left(k_{l}\right)}}} .
$$

Из (14) также получаем

$$
\begin{aligned}
& \left(\begin{array}{cc}
\beta_{0}(r) & -\beta_{1}(r) \\
\beta_{1}(r) & \beta_{0}(r)
\end{array}\right)=\prod_{j=1}^{r}\left(\left(\begin{array}{cc}
b_{0}^{(j)} & -b_{1}^{(j)} \\
n_{j}(j) & b_{0}^{(j)}
\end{array}\right)+\left(\begin{array}{cc}
\theta_{0}^{(j)} & -\theta_{1}^{(j)} \\
b_{1}^{(j)} & \theta_{0}^{(j)}
\end{array}\right)\right)= \\
& =\prod_{j=1}^{r} n_{j}\left(\begin{array}{cc}
b_{0}^{(j)} & -b_{1}^{(j)} \\
b_{1}^{(j)} & b_{0}^{(j)}
\end{array}\right)\left(I+\sum_{k=1}^{r} \frac{1}{\sqrt{n_{k}}}\left(\begin{array}{cc}
\widetilde{\theta}_{0}^{(k)} & -\widetilde{\theta}_{1}^{(k)} \\
\widetilde{\theta}_{1}^{(k)} & \widetilde{\theta}_{0}^{(k)}
\end{array}\right)\left(\begin{array}{cc}
b_{0}^{(k)} & -b_{1}^{(k)} \\
b_{1}^{(k)} & b_{0}^{(k)}
\end{array}\right)^{-1}+W^{\prime}\right)
\end{aligned}
$$

(мы воспользовались тем, что все матрицы в разложении коммутативны), где I - единичная матрица, а

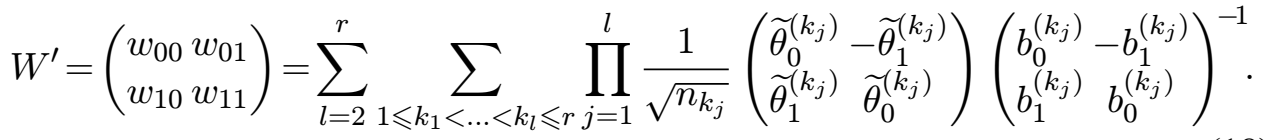


Из (14) - (18) следует равенство

$$
\left(\begin{array}{ccc}
\beta(r) & 0 & 0 \\
0 & \beta_{0}(r) & -\beta_{1}(r) \\
0 & \beta_{1}(r) & \beta_{0}(r)
\end{array}\right)=\prod_{j=1}^{r} n_{j} B^{(j)}\left(I+\sum_{k=1}^{r} \frac{1}{\sqrt{n_{k}}} D^{(k)}\left(B^{(k)}\right)^{-1}+W\right)
$$

где

$$
D^{(k)}=\left(\begin{array}{ccc}
\widetilde{\theta}^{(j)} & 0 & 0 \\
0 & \widetilde{\theta}_{0}^{(j)} & -\widetilde{\theta}_{1}^{(j)} \\
0 & \widetilde{\theta}_{1}^{(j)} & \widetilde{\theta}_{0}^{(j)}
\end{array}\right), \quad W=\left(\begin{array}{ccc}
w & 0 & 0 \\
0 & w_{00} & w_{01} \\
0 & w_{10} & w_{11}
\end{array}\right)
$$

Так как векторы $\widetilde{\Theta}^{(l)}$ и $\widetilde{\Theta}^{(k)}$ независимы при $l \neq k$, а математические ожидания их компонент равны нулю, то из формул (19) и (20) следует равенство (3). Теорема 1 доказана.

Перейдем к доказательству теоремы 2. Из определений, неравенств $n_{1}<$ $n_{2}<\ldots<n_{r}$ и леммы 1 (см. замечание 2) вытекает, что $W=O\left(n_{1}^{-1}\right)$. Поэтому предельные распределения случайных матриц

$$
\begin{gathered}
\sqrt{n_{1}}\left(\frac{1}{n_{1} n_{2} \cdots n_{r}}\left(\begin{array}{ccc}
\beta(r) & 0 & 0 \\
0 & \beta_{0}(r) & -\beta_{1}(r) \\
0 & \beta_{1}(r) & \beta_{0}(r)
\end{array}\right)-\mathbb{B}(r)\right), \\
\sum_{j=1}^{r} \frac{\sqrt{n_{1}}}{\sqrt{n_{j}}} \mathbb{B}(r) D^{(j)}\left(B^{(j)}\right)^{-1}
\end{gathered}
$$

совпадают, если хотя бы одно из них существует.

Более того, в силу условия $n_{1} / n_{j} \rightarrow \rho_{j}$ вторую матрицу здесь можно заменить матрицей

$$
H=\left(\begin{array}{ccc}
h & 0 & 0 \\
0 & h_{0} & -h_{1} \\
0 & h_{1} & h_{0}
\end{array}\right)=\sum_{j=1}^{r} \rho_{j} \mathbb{B}(r) D^{(j)}\left(B^{(j)}\right)^{-1}
$$

Сказанное означает, что далее можно ограничиться изучением асимптотического поведения распределения матрицы $H$. 
Для $h, h_{0}, h_{1}$ получаем выражения

$$
\begin{gathered}
h=b^{*} \sum_{j=1}^{r} \rho_{j} \frac{\widetilde{\theta}^{(j)}}{b^{(j)}}, \\
h_{0}=b_{0}^{*} \sum_{j=1}^{r} \rho_{j} \frac{\widetilde{\theta}_{0}^{(j)} b_{0}^{(j)}+\widetilde{\theta}_{1}^{(j)} b_{1}^{(j)}}{\left(b_{0}^{(j)}\right)^{2}+\left(b_{1}^{(j)}\right)^{2}}+b_{1}^{*} \sum_{j=1}^{r} \rho_{j} \frac{\widetilde{\theta}_{0}^{(j)} b_{1}^{(j)}-\widetilde{\theta}_{1}^{(j)} b_{0}^{(j)}}{\left(b_{0}^{(j)}\right)^{2}+\left(b_{1}^{(j)}\right)^{2}}, \\
h_{1}=b_{1}^{*} \sum_{j=1}^{r} \rho_{j} \frac{\widetilde{\theta}_{0}^{(j)} b_{0}^{(j)}+\widetilde{\theta}_{1}^{(j)} b_{1}^{(j)}}{\left(b_{0}^{(j)}\right)^{2}+\left(b_{1}^{(j)}\right)^{2}}-b_{0}^{*} \sum_{j=1}^{r} \rho_{j} \frac{\widetilde{\theta}_{0}^{(j)} b_{1}^{(j)}-\widetilde{\theta}_{1}^{(j)} b_{0}^{(j)}}{\left(b_{0}^{(j)}\right)^{2}+\left(b_{1}^{(j)}\right)^{2}} .
\end{gathered}
$$

Отсюда вытекает равенство

$$
\left(\begin{array}{l}
h \\
h_{0} \\
h_{1}
\end{array}\right)=\sum_{j=1}^{r} \rho_{j} V^{(j)} \widetilde{\Theta},
$$

где $V^{j}$ - матрицы, введенные формулой (5).

Из леммы 1 и (22) следует, что предельное распределение вектора $\left(h, h_{0}, h_{1}\right)^{T}$ совпадает с распределением вектора

$$
U(r)=\sum_{j=1}^{r} \rho_{j} V^{(j)}\left(\begin{array}{c}
\eta^{(j)} \\
\eta_{0}^{(j)} \\
\eta_{1}^{(j)}
\end{array}\right),
$$

где $\left(\eta^{(j)}, \eta_{0}^{(j)}, \eta_{1}^{(j)}\right)^{T}$ - независимые случайные векторы, имеющие нормальные распределения с нулевым вектором средних и матрицами ковариаций $\widetilde{\Sigma}^{(j)}$. Используя известные свойства матриц ковариаций (см. [6, с. 329]), получаем, что $U(r)$ имеет нормальное распределение с нулевым вектором средних и ковариационной матрицей

$$
\sum_{j=1}^{r} \rho_{j}^{2} V^{(j)} \widetilde{\Sigma}^{(j)}\left(V^{(j)}\right)^{T} .
$$

В силу изложенного выше такое же предельное распределение имеет и вектор $\nabla(r)$. Теорема 2 доказана.

Авторы благодарны А. М. Зубкову и А. М. Шойтову за полезные замечания.

2016, T. 7, № 4, С. 81-94 


\section{Список литературы}

[1] Pohl P., "Description of MCV, a pseudo-random generator", Scand. Actuarial. J. (1976), 1-14.

[2] Меженная Н. М., Михайлов В. Г., “О распределении числа единиц в выходной последовательности генератора Пола над полем $G F(2)$ ", Математические вопросы криптографии, $4: 4$ (2014), 51-58.

[3] Меженная Н. М., “О распределении числа единиц в двоичной мультициклической последовательности”, Прикладная дискретная математика, № 1 (2015), 69-77.

[4] Mezhennaya N. M., "Convergence rate estimators for the number of ones in outcome sequence of MCV generator with m-dependent registers items", Siberian Electronic Mathematical Reports, 11 (2014), 18-25.

[5] Меженная Н. М., Михайлов В. Г., “О числе появлений знаков в мультициклической случайной последовательности по модулю 4”, Дискретная математика, 26 :4 (2014), 51-58.

[6] Крамер Г., Математические методы статистики, М.: Мир, 1975. 